\title{
1. EVOLUCIÓN DE LOS CONCEPTOS SOBRE inteligencia. Planteamientos actuales DE LA INTELIGENCIA EMOCIONAL PARA LA ORIENTACIÓN EDUCATIVA
}

Purificación Salmerón Vílchez

Universidad de Granada

\section{INTRODUCCIÓN}

Los planteamientos constructivistas de la LOGSE, a mi parecer, se han interpretado medianamente en el ámbito educativo. Se han considerado como una teoría sobre el aprendizaje y la enseñanza que, en el ámbito cognitivo, podría contener las respuestas a cuantos problemas se planteasen en la educación. Eso no es del todo cierto.

Para el tema que vamos a desarrollar, nos interesa más resaltar las afirmaciones que en este aspecto hace Delval, J. (1990): Lo más original del constructivismo es que se trata de una teoría epistemológica que trata de explicar la formación del conocimiento situándose en el interior de la persona que aprende.

Ese interior que para unos es el ámbito cognitivo, para otros es una conjunción de cognición y sentimiento. Personalmente me adhiero a este segundo grupo.

En este sentido, y antes de abordar el objeto específico de este trabajo, considero necesario realizar una serie de cuestionamientos que justifican la necesidad de abordar este tema. Cada uno de los que lo lean podrían hacer 
éstas y muchas más. A mí, la reflexión y lectura de los trabajos sobre inteligencia me han sugerido las siguientes:

- ¿Qué papel desempeñan los sentimientos en la actividad mental o el desarrollo cognitivo de las personas?

- ¿Cómo interviene la experiencia emocional en la madurez intelectual?

- ¿Cuál es conjunto de habilidades-capacidades que las personas deberían identificar y desarrollar en su contexto para que les vaya bien en la vida?

- Si se desarrollasen esas capacidades en el currículum escolar ¿ampliaríamos las oportunidades de éxito de los alumnos? ¿Mejoraríamos su rendimiento intelectual y académico? ¿Evitaríamos la aparición de riesgos?

- ¿Cómo diseñar y aplicar programas de desarrollo de inteligencia emocional en contextos donde coexisten modelos de vida en los que sentimientos y emociones positivas se mezclan y han de interaccionar con otros no tan positivos donde el egoísmo, la violencia, la mezquindad, proporcionan más ventajas económicas que los primeros?

No es mi cometido ahora dar respuesta a todas, aunque si intentaré aportar elementos para comprender la inteligencia desde planteamientos actuales no apoyados exclusivamente en fundamentos de psicología cognitiva, al mismo tiempo que aportar resultados en cuanto a efectos de programas educativos implementados bajo los principios de las inteligencias múltiples de Gadner (1996).

Apoyándonos en los planteamientos de desarrollo de lo que se viene denominando inteligencia emocional, organizaré, mediante un sistema de indicadores, las dimensiones, elementos, indicadores y variables que a nivel general habrían de tenerse en cuenta en el diseño genérico de una intervención educativa, aportando también instrumentación para el diagnóstico de aspectos relacionados con la inteligencia emocional.

\section{EL RECORRIDO DE LA INVESTIGACIÓN SOBRE INTELIGENCIA}

No ha existido ni existe univocidad en el concepto de inteligencia, aunque detrás de las diferentes concepciones existen planteamientos generales teóricos que de alguna manera les dan sentido y validez. 
Un aspecto curioso de las investigaciones sobre la inteligencia es que, conforme han ido evolucionando las concepciones de la misma, paralelamente a cada una de ellas ha surgido y se ha impuesto más la necesidad de medirla que validar los constructos teóricos de partida, generándose más investigaciones sobre la instrumentación para medirla que sobre la conceptualización.

La búsqueda de los conceptos de inteligencia ha seguido las pautas marcadas por los investigadores que querían medirla. Hasta la década de $\mathbf{1 9 7 0}$ parece que lo único que interesa a los investigadores de la inteligencia es su componente cognitivo. La justificación de esta tendencia estaría en la peculiar historia del desarrollo de la psicología que, en el campo que estamos tratando, sólo ha sido rigurosa en la investigación sobre construcción de tests, pero no siempre en la validación científica de los constructos teóricos de los que partía ni en la aplicación correcta de estas investigaciones. De ahí que el diagnóstico de la inteligencia haya pasado por fases hasta de rechazo social tan alto como para que en algunos estados de América del Norte se prohibiera administrar a los niños test de inteligencia en las escuelas o se produjeran quemas públicas de esos tests en plazas de algunas ciudades.

A partir de los intereses de los investigadores, podríamos establecer la siguiente clasificación de modelos de inteligencia:

A) Modelos centrados en la estructuración-composición de la inteligencia. El interés ha sido la búsqueda del factor o factores que componen-dominan en el constructo inteligencia, sus relaciones, identificarlos para medirlos, establecer sus relaciones y, en base a esas medidas y relaciones, poder describir diferencias interindividuales.

B) Modelos centrados en el funcionamiento cognitivo de la inteligencia. Su interés es el conocimiento de los procesos mentales que dirigen las acciones para intervenir modificando cognitivamente las estructuras, de manera que la medición favorezca otras estructuras más apropiadas y más complejas que permitan mayor autonomía a las personas en el aprendizaje y el conocimiento.

C) Modelos centrados en la comprensión global del desenvolvimiento social de las personas en la búsqueda de su felicidad como necesidad vital. Ello conlleva necesariamente la consideración de que el funcionamiento de las personas en sociedad se produce mediante cognición y sentimiento, predominando en algunas situaciones comportamentales otras dimensiones diferentes a la cognición. 


\subsection{MODELOS CENTRADOS EN LA ESTRUCTURACIÓN- COMPOSICIÓN DE LA INTELIGENCIA}

\section{* Inteligencia monolítica}

Desde que el equipo Binet-Simón, psicólogo y pedagogo franceses, recibieran de los responsables de la Administración francesa el encargo de disenar el modo de cumplir con el principio de igualdad en las escuelas, hasta nuestros días, el mayor interés de la investigación ha estado en identificar las dimensiones o aspectos fundamentales de la inteligencia.

Binet (1904) partió del siguiente supuesto teórico: la inteligencia se manifiesta en la rapidez de aprendizaje (por lo menos de aprendizajes a largo plazo). A partir de ahí trató de elaborar pruebas que identifiquen la rapidez con que aprende un niño normal. Estas pruebas estarían constituidas por conocimientos que se corresponden a cada edad en situaciones de normalidad. Estos conocimientos constituyeron los items de la escala para medir la inteligencia.

La consecuencia lógica de este planteamiento fue que se puede medir la capacidad intelectual a partir del nivel de conocimientos que se muestra en un momento dado.

El concepto fundamental para Binet fue la Edad mental como la edad que se corresponde con las respuestas correctas que una persona da al contestar su escala.

La clasificación de la persona como inteligente normal, superior o inferior vendría dada según que el sujeto contestase bien a los items de conocimiento que le correspondían a su Edad cronológica, a los de mayor o a los de menor edad que él.

La intención era discriminar los débiles mentales. Si la inteligencia los discriminaba, entonces es que podía ser medible.

Para medirla tenía en cuenta diferentes funciones como la memoria, fantasía, imaginación, atención, comprensión, sugestibilidad, apreciación estética, sentimiento moral, abstracción, pensamientos sin imágenes, tiempo de reacción, etc...

La concepción de la inteligencia que respalda a estas pruebas es multidimensional, basada en diferentes aptitudes; sin embargo cuando se le mide e interpreta, se hace como si de una sola variable se tratara (edad mental).

La influencia de los estudios de Binet-Simon se produjo de forma relevante en los instrumentos desarrollados con posterioridad dentro de la concepción monolítica de la inteligencia, entre los que resalta la adaptación ameri- 
cana: El Standford-Binet de Terman (1916) y las versiones posteriores (Standford-Binet, 1960) transformando el concepto de coeficiente intelectual en una medida de dispersión, pero con variaciones esencialmente de tipo psicométrico.

Otro estudio relevante, que demuestra la poca rigurosidad de los constructos teóricos de partida, es el de Spearman que apoyándose en procedimientos matemáticos de análisis multivariante, en su caso la metodología del análisis factorial, genera la Teoría del Factor. De los tests de inteligencia analizados por este autor, él destaca que todos miden en su mayor parte un factor general "g", que es la inteligencia propiamente dicha, y otro específico «s», que aparece en menor grado y que corresponde a las características de cada test. O sea, que el factor principal "g» estaría implicado en toda actividad intelectual y, por consiguiente, estaría contenido en todos los items y en todos los tests intelectuales. El factor "ऽ" sólo estaría relacionado con la tarea concreta que constituyera cada ítem y por tanto dependiente de otras destrezas específicas no intelectuales.

Aunque ambos, Binet y Spearman, estudian la inteligencia desde esta concepción monolítica, al medirla son radicalmente distintos.

Binet está motivado por aplicar en los entornos escolares sus hallazgos. A Spearman sólo le interesan los resultados brutos que obtienen al utilizar la metodología del análisis factorial, forzando su significación psicológica.

\section{* Los planteamientos factorialistas}

Casi todos se desarrollan en Estados Unidos y parten de la visión de un intelecto compuesto.

Los estudios de Spearman generan una reacción en cadena que intenta mejorar los procesos del análisis factorial (Genovard Roselló, C. y Castelló Tarrida, A., 1990).

Este es el caso de Thurnstone (1938). Partiendo de los trabajos de Spearman, llega a un modelo de inteligencia factorializada, o sea, constituida por una serie de componentes básicos esencialmente independientes entre ellos, pero cuyo efecto combinado equivale al rendimiento intelectual.

Las causas de este otro concepto de inteligencia, utilizando el mismo procedimiento matemático del análisis factorial, estarían en que Thurstone fuerza la metodología matemática de análisis de datos para que se ajuste a los presupuestos teóricos propios de los contenidos psicológicos de las variables implícitas en cada ítem, buscando que la correlación estadística que aparezca esté fundamentada por una sólida correlación conceptual anterior. 
Los resultados no eran concordantes con el modelo de Spearman: no se observaba la existencia de ningún factor general y dominante y sí aparecían una serie de aptitudes específicas, independientes entre sí.

Esta estructura factorial provoca otra concepción de la inteligencia, al mismo tiempo que otra forma de medirla.

Más tarde Eysenck, estudiando a Thurstone, llega a la conclusión de que matemáticamente se pueden reorganizar los factores alrededor de un posible factor "g" de Spearman y otros factores específicos (aptitudes) muy parecidos a los de Thurstone.

Es curioso que tantas variaciones sobre el concepto de la inteligencia, desarrollado hasta los años sesenta, haya tenido como origen la metodología de análisis de los datos extraídos de los tests de inteligencia producidos. Parece como si la intención de medirla fuese independiente de las concepciones teóricas de la misma.

Así, podemos observar tres interpretaciones diferentes de unos datos casi idénticos:

- Spearman se centra en el factor general y desprecia la significación de los factores específicos.

- Thurstone redistribuye el efecto conjunto de G y $\mathbf{S}$ en sus aptitudes mentales primarias como factores independientes.

- Eysenck respeta el valor de G y analiza la factorialización de "S» llegando a una solución intermedia entre los anteriores autores.

Es Guilford (1967) quien genera la necesidad de estructurar y categorizar los cuantiosos factores detectados en las diferentes investigaciones factoriales de las décadas 40 y 50 .

Crea un modelo, "estructura del intelecto", que responde a un intento de catalogación de los factores, aportando al mismo tiempo un marco de referencia amplio para la interpretación de los mismos.

\section{* Una concepción jerárquica de los factores}

Si por un lado las concepciones monolíticas defienden una inteligencia unitaria y los factorialistas una inteligencia compuesta, los autores de planteamientos jerárquicos tienen sus raíces en ambas concepciones, evolucionando a partir de los modelos factoriales empleados.

Varían de sus predecesores en la forma de análisis de los datos, en la consideración del factor "g" como culminación de la jerarquía y en la valoración de los diferentes factores de la estructura jerárquica. 
La metodología utilizada en todos los casos es el análisis factorial, por lo que los diferentes trabajos conducen a la identificación de factores. Unos tienden a incluir en el nivel superior de la jerarquía de factores "g" de Spearman (Burt, Vernon, Catell) mientras que otros prescindieron de él (Jäger, 1967).

La diferencia con los factorialistas estaría en que éstos valoran y definen componentes equivalentes en cuanto a la correlación estadística y ordenación, y los jerárquicos se refieren a factores de mayor importancia o generalidad y a subdivisiones de factores.

De entre los investigadores resalta Catell, que tomando los trabajos de Spearman, continúa la línea de Thurstone, aunque partiendo de factores primarios distintos: aquéllos que se habían manifestado como relativamente estables.

\subsection{MODELOS CENTRADOS EN EL FUNCIONAMIENTO COGNITIVO DE LA INTELIGENCIA}

El interés ya no está en la identificación, definición de las variables o dimensiones del comportamiento inteligente, sino que se centra en cómo evoluciona y se desarrolla dicha estructura. En los efectos sobre la misma de la herencia o influencia del ambiente. Interesa más lo cualitativo que lo cuantitativo en el análisis de la estructura de la inteligencia.

La investigación psicológica estará influida por lo que se ha venido en llamar la revolución cognitiva.

El centro de atención de la ciencia psicológica se dirige a averiguar la forma en que la mente registra, almacena, procesa información y cuál es su naturaleza.

Destacan Piaget, Vigotsky, Brunner, Wallon, entre los enfoques evolutivos, y Eysenck, White, Catell, Vernon, Jensen... entre los enfoques cualitativos.

Resaltan para la educación los trabajos de Piaget y Vigotsky. Piaget interesado en el desarrollo de las formas de conocimiento del niño y su preocupación por el origen de la inteligencia: biológico (percepción y motricidad) y lógico (considera las relaciones lógicas y matemáticas como irreductibles); por tanto, el análisis de las funciones intelectuales superiores dependerá del análisis de dichas relaciones.

Vigotsky se interesa más en el desarrollo potencial de la inteligencia. Para este autor el desarrollo humano se basa en dos procesos: la maduración y el aprendizaje. El aprendizaje humano presupone una naturaleza social específica y un proceso mediante el cual los niños acceden a la vida intelectual de aquéllos que les rodean. 
La inteligencia es, en este sentido, un producto social. La zona de desarrollo potencial será el conjunto de actividades que el niño es capaz de realizar con la ayuda y colaboración de las personas que le rodean.

Los conceptos más representativos del nivel de desarrollo potencial son:

a) Relación entre aprendizaje y desarrollo. El aprendizaje crea la zona de desarrollo potencial y, como tal, el proceso de desarrollo es una consecuencia de aquél.

b) El desarrollo de las funciones humanas superiores e intelectuales es necesariamente artificial. Es un artificio y un producto de la cultura y de las relaciones con los demás. La zona de desarrollo potencial se debe a la influencia activa del mediador y al aprendizaje activo del sujeto.

c) Los test de inteligencia tradicionales nos indican la zona de desarrollo real, pero no sus potenciales.

\subsection{MODELOS CENTRADOS EN LA COMPRENSIÓN GLOBAL DE LA PERSONA PARA UN MEJOR DESARROLLO DE SU VIDA}

\section{Las inteligencias múltiples}

El desarrollo armónico de la vida de las personas, el éxito social, académico, afectivo, profesional.... no puede ser explicado sólo a partir de una concepción monolítica, factorial de la inteligencia. Ni tampoco con el sólo funcionamiento de una adecuada estructura cognitiva para analizar o ejecutar cualquier realidad técnicamente bien. No hay realidad que, al ser conceptualizada por la mente humana, no vaya acompañada por la asignación de un valor o un sentido que le otorga el que la conceptualiza.

Muchos comportamientos, capacidades, consideradas excepcionales, no son producto exclusivo de la cognición. Las personas son capaces de operar inteligentemente en facetas que tienen componentes de muy diverso matiz.

Gadner (1989) introduce su modelo de inteligencia múltiple, en el que considera las siguientes variedades distintas de inteligencia:

- Inteligencia académica determinada por la capacidad verbal y la aptitud lógico matemática.

- La capacidad espacial (arquitectos, artistas en general).

- La inteligencia kinestésica (capacidad para la utilización del curso). 
- La inteligencia musical.

- Las inteligencias interpersonales.

- La inteligencia intrapsíquica o intrapersonal.

A su vez, la inteligencia interpersonal la subdivide en cuatro capacidades diferentes: liderazgo, aptitud de establecer relaciones, mantener amistades, capacidad de solucionar conflictos y capacidad para el análisis social.

En trabajos posteriores de este autor, Gardner, H. (1993) distingue entre inteligencia interpersonal e inteligencia intrapersonal. Define a la inteligencia interpersonal como la capacidad de comprender a los demás. Afirma que las personas que la han desarrollado son capaces de discernir y responder apropiadamente a los estados de ánimo, temperamentos, motivaciones y deseos de las demás personas. La intrapersonal sería una habilidad correlativa del interior que nos permite configurar una imagen exacta y verdadera de nosotros mismos y que nos hace capaces de utilizar esa imagen para actuar en la vida de un modo más eficaz.

El modelo cognitivo que aún prevalece constituye una visión reduccionista del funcionamiento mental, que no acaba por explicar la función de los sentimientos en la vida intelectual, ignorando muchas de las facetas humanas que no dependen de la lógica de la razón intelectual, sino de los sentimientos que acompañan a la vida y que determinan más exactamente la cualidad de la información que recibimos. Sin embargo, algo está cambiando en la Psicología actual.

El mismo Gadner, en el que predomina una perspectiva cognitiva de la inteligencia, admite que, cuando escribía por primera vez sobre las inteligencias personales, se refería a las emociones, en especial cuando se refería a la inteligencia intrapersonal (Goleman, D., p. 77).

En los últimos años, cada vez son más los trabajos de psicólogos (Stemberg, R. J. 1985, Salovey, P. y Mayer, J.D. 1990) que manifiestan la necesidad de reformular el concepto de inteligencia en términos de aquello que hace que una persona enfoque más adecuadamente su vida. Así el concepto de inteligencia está más cerca de lo personal o emocional.

\section{La inteligencia emocional}

Los trabajos de Gadner dieron un nuevo sentido al concepto tradicional de la inteligencia. La educación para la vida, hoy, necesita desarrollar en las personas más dimensiones que la capacidad de abstracción, la lógica formal, la comprensión de complejas implicaciones o amplios y precisos conocimientos. Es necesario incorporar el desarrollo de otras, como la creatividad, la capacidad de organización, de relación, de motivación, de actitudes positivas hacia 
los demás, etc. variables estas que han estado ligadas a rasgos del constructo "personalidad» o "carácter», pero que hoy se las considera más ligadas al funcionamiento emocional del cerebro (Martín, D. y Boek, K. 1997).

Es Salowey (1990) quien, apoyándose en los trabajos de Gadner, desarrolla el concepto de Inteligencia Emocional; constructo que estaría constituido por cinco dimensiones principales:

- El conocimiento de las propias emociones como la capacidad de reconocer un sentimiento en el mismo momento en que aparece, al mismo tiempo que las causas y sus efectos.

- La capacidad de controlar las emociones para adecuarlas a cada momento sin que nos veamos arrastrados por ellas.

- La capacidad de motivarse a sí mismo, utilizando lo mejor de lo que tenemos para aprender a disfrutar con y en las propias tareas que hacemos, sin que tengan que influir refuerzos externos a la tarea o a nosotros mismos. La satisfacción del rendimiento induce a rendir más.

- El reconocimiento de las emociones ajenas como la capacidad empática que posibilita la predisposición de admitir las emociones de los demás, a escuchar y comprender desde la perspectiva del otro así como a identificar y comprender sentimientos ajenos, aunque no se haya expresado verbalmente.

- Capacidad para controlar las relaciones y hacerlas adecuadas con los demás a cada momento.

El aspecto más importante es la consideración de estas dimensiones como habilidades innatas en todas las personas, en mayor o menor grado y en diferente cualidad. Lo importante es su consideración dinámica y, por tanto, la posibilidad de desarrollarlas adecuadamente por una mediación educativa.

Una persona emocionalmente desarrollada es aquélla que gobierna adecuadamente sus sentimientos, al mismo tiempo que sabe interpretar y relacionarse eficaz y eficientemente con los sentimientos de los demás, buscando éxito y productividad, y sintiendo satisfacción con todo ello.

\section{COMPETENCIAS DE UNA INTELIGENCIA EMOCIONAL DESARROLLADA}

Siguiendo a Salovey, P. (1990), consistirían en:

- Reconocimiento de uno mismo: capacidad de reconocimiento de las propias emociones en el mismo momento en que aparecen. También 
podría interpretarse por la percepción adecuada en el tiempo y espacio que nos alerta de nuestros verdaderos sentimientos.

- Control de sí mismo: capacidad de adecuación de los sentimientos al momento, así como la capacidad de recuperarse de contratiempos.

- Dirigir el control hacia metas planificadas: capacidad de control de la vida emocional y subordinarla a un objetivo, o la capacidad de motivarse adecuadamente a uno mismo.

- Reconocimiento de lo ajeno: Capacidad para controlar adecuadamente las relaciones con las emociones ajenas.

Cada una de estas competencias o dominios representa un conjunto de habilidades, hábitos y estrategias que, con una mediación educativa adecuada, pueden mejorar el desarrollo personal, académico y social de los alumnos.

\subsection{EL CONOCIMIENTO DE LAS PROPIAS EMOCIONES}

El conocimiento de uno mismo supone al menos:

- Reconocer cuándo se está atrapado por un sentimiento que puede arrastrarle a conductas no deseadas socialmente.

- Percibir e identificar los sentimientos propios en el mismo momento en que éstos se dan.

- Experimentar y comunicar el sentimiento en el momento oportuno. En ocasiones lo hacemos a destiempo y nos arrepentimos de haberlo hecho así.

La conciencia de uno mismo podría interpretarse como la atención continua dirigida a los estados y dinámica de los sentimientos propios. Es, como apuntan Kabat-Zinn, J. (1994), la conciencia autorreflexiva en la que la mente se ocupa de observar e investigar la experiencia misma incluidas las emociones.

Es un posicionamiento de actividad no reactiva, neutral, como si de un testigo se tratara, que activa, dirige y mantiene la atención sobre uno mismo.

A modo de ejemplo: una persona con esta capacidad desarrollada puede al mismo tiempo estar discutiendo violentamente con alguien y tener la conciencia autorreflexiva de que está discutiendo.

Por tanto, se trata de ser consciente de los propios estados de ánimo y de los pensamientos que se tienen de esos estados de ánimo. 
Posibles tipologías de personas que hayan desarrollado esta capacidad serían:

* Personas conscientes de si mismas

Son conscientes de sus estados de ánimo mientras los están experimentando: autónomas, seguras, tienen visión positiva de la vida, no se obsesionan ante un estado de ánimo negativo y salen pronto de él.

* Personas atrapadas por sus emociones

Están desbordadas por sus emociones y son incapaces de escapar de ellas. Son esclavas de sus estados de ánimo. Se sienten incapaces de controlar su vida emocional y, por tanto, no tratan de escapar de los estados de ánimo negativos.

* Personas que aceptan resignadamente sus emociones

Perciben con claridad lo que están sintiendo. Aceptan pasivamente sus estados de ánimo y no suelen tratar de cambiarlos: «resignación con la situación en que se encuentran".

\subsection{EL CONTROL DE LAS EMOCIONES}

Se trataría de la capacidad de gobernar y dirigir al menos los siguientes aspectos:

* La contención del exceso emocional: el equilibrio, lo apropiado. «Cada sentimiento es válido y tiene su propio valor y significado».

* El aprendizaje preciso para mantenerse en equilibrio emocional.

* La integración de acciones eficaces para ejercitar la calma de uno mismo.

Es difícil controlar el momento en que nos vemos arrastrados por una emoción y tampoco podemos hacer mucho sobre el tipo de emoción que nos llegará, pero sí podemos controlar el tiempo que permanecerá una determinada emoción.

\subsubsection{Indicadores de la capacidad de control de las emociones}

* La autorregulación emocional. Se trataría de saber reconocer en qué momento la excitación crónica del cerebro emocional es tan intensa como para requerir tratamiento externo, terapéutico o farmacológico.

* Inexistencia-existencia de necesidad de cambiar el estado de ánimo. 
* Creencia en la necesidad de crearse determinados estados de ánimo para utilizarlos en la práctica con intención de obtener mejores resultados.

* Manipulación de los propios estados de ánimo para fines predeterminados.

\subsection{LA CAPACIDAD DE MOTIVARSE A UNO MISMO}

La motivación interna está ligada a sentimientos tales como el entusiasmo, la perseverancia, confianza en sí mismo, satisfacción personal en el ejercicio de la actividad, etc.

A su vez, la perseverancia depende de factores emocionales tales como el entusiasmo y la tenacidad frente a todo tipo de contratiempos, una adecuada educación para el trabajo...

Una motivación de logro bien relacionada con un bajo temor al fracaso, acompañada de satisfacción de las tareas necesarias para el logro de metas, provoca el grado óptimo emocional para mantenerse en procesos de consecución de metas a largo plazo.

\subsubsection{Indicadores de esta capacidad}

* El control de los impulsos, o la capacidad de demorar los impulsos que permite llevar a cabo una gran cantidad de actividades mientras se espera la gratificación deseada.

Esta capacidad ha de ser interpretada como capacidad intelectual de represión de los propios impulsos al servicio de una meta y, por lo tanto, como un acto esencial de autorregulación emocional.

* Capacidad de pensamiento positivo. Capacidad de dirigir el ánimo hacia estados de ánimo que aumentan la facilidad de pensar con flexibilidad y complicidad, haciendo más fácil encontrar soluciones a problemas de todo tipo.

* La esperanza o creencia en que se dispone de voluntad y modo de conseguir las metas que se propone.

* El optimismo realista, o actitud emocionalmente inteligente, por el que se dispone de un alto nivel de expectativas de que las cosas irán bien a pesar de todo.

* La autoeficacia o creencia de que se tiene el control de los acontecimientos de su vida y la capacidad para hacer frente a los problemas a medida que se presentan. 
* El rendimiento máximo, grado superior de control de las emociones al servicio del rendimiento y el aprendizaje.

La atención se focaliza tanto que la persona sólo es consciente de la estrecha franja de percepción relacionada con la tarea que está llevando a cabo. La persona se absorbe por completo en lo que está haciendo y su conciencia se funde con la acción.

La motivación a hacer una tarea cada vez mejor está en permanecer en rendimiento máximo mientras se lleva a cabo.

\subsection{CAPACIDAD DE RECONOCER LAS EMOCIONES AJENAS: LA EMPATÍA}

La conciencia de sí mismo es la capacidad sobre la que se erige la empatía, puesto que cuanto más estemos abiertos a nuestras emociones, mayor será nuestra habilidad para comprender los sentimientos de los demás.

Es imprescindible una formación en la interpretación de mensajes por vía no verbal, ya que no es frecuente que las personas manifiesten sus emociones oralmente.

La empatía exige estados de calma y receptividad suficientes para que las señales, sutiles, manifestadas por los sentimientos de la otra persona puedan ser captadas y reproducidas por nuestro propio cerebro emocional.

\subsection{EL CONTROL DE LAS RELACIONES: LA COMPETENCIA SOCIAL O INTELIGENCIA INTERPERSONAL}

Para llegar a ser competente en el control de las emociones de los demás y dominar el ámbito de las relaciones es preciso haber desarrollado el autocontrol y la empatía.

La carencia de estas habilidades es la causa de que hasta las personas intelectualmente más brillantes, desde una perspectiva cognitiva, fracasen en sus relaciones y resulten en ocasiones arrogantes, insensibles y hasta odiosas.

Los indicadores de esta capacidad serían:

- Alto nivel en la organización de grupos.

- Facilidad en la generación y desarrollo de procesos para negociar soluciones. 
- Alto nivel en el establecimiento de conexiones personales.

- Sensibilidad social, o gran habilidad, para el análisis social y la identificación rápida de las situaciones y roles que ocurren en su alrededor.

\section{INSTRUMENTOS PARA LA APRECIACIÓN DE LAS EMOCIONES}

Tras la publicación de Daniel Goleman (1995), se están sucediendo publicaciones en las que se percibe la intención, no muy acertada, de divulgar procedimientos de medición de la inteligencia emocional, utilizando el cuestionario como instrumento (Martineaud, S. y Engelhart, D., 1997; Martin, D. y Boeck, K., 1997; Torrabadella, P., 1997).

El objeto de todos es facilitar al lector una forma de autoanálisis de las capacidades que hemos descrito en el apartado anterior, aunque sin aportar justificación ni características técnicas de los cuestionarios que presentan.

El mismo Goleman, en su exhaustiva revisión sobre la inteligencia emocional, no sólo no aporta instrumentación para medirla, sino que advierte, y muy acertadamente, la gran dificultad de medir la variabilidad interpersonal de la inteligencia emocional.

Por tanto, habrá que vigilar y ser cauteloso a la hora de identificar y apreciar aspectos de inteligencia emocional si no es con instrumentación que haya sido construida y validada científicamente para ese efecto. Los tests existentes, incluso el introducido por el autor de "la inteligencia emocional" en Internet, habrían de utilizarse, si es por curiosidad no científica, como meros juegos de acercamiento al constructo teórico que estamos analizando.

Hasta que se construyan y disponga de instrumentos científicos, bajo las nuevas concepciones, sólo podemos aportar algunos que, en forma de escalas de medición, tienden a apreciar estados afectivos y factores reguladores de éstos, aclarando que en los constructos que miden subyace la concepción cognitiva de la inteligencia en los investigadores constructores de dichos tests, con lo cual, y a nivel teórico, dichos tests no miden el mismo constructo analizado desde la perspectiva emocional de la psicología. Aclarado esto, a continuación se describen brevemente unos cuantos test aportados por Vergara, A. y otros (1989) relativos a estados de ánimo positivos o negativos, autoconciencia, deseabilidad social, ansiedad y reacciones emocionales, de control de emociones.

- Escala de adjetivos afectivos negativos y positivos de Diener y al. (1985). Esta escala pretende obtener una medición simple, pero fiable, de los estados afectivos de las personas. 
- Escala de adjetivos múltiples revisada por Gotlib y Meyer (1986). Busca medir tres estados de ánimo: hostilidad, ansiedad y depresión.

- Escala de intensidad afectiva de Larsen y al. (1986). Pretende medir la reacción afectiva ante los sucesos y situaciones emocionales.

- Escala de autoconciencia pública y privada de Scheier y Carver (1985). Mide la disposición o tendencia de las personas a focalizar su atención sobre sí mismos, ya sea sobre sus aspectos privados (sentimientos, estados de ánimo, pensamientos, etc.) o sobre sus aspectos públicos (comportamiento externo, aspecto físico, etc.).

- La combinación de las escalas de deseabilidad social de Crowne-Marlowe y la escala de ansiedad de Taylor para estudiar reacciones emocionales.

La escala de deseabilidad social de Crowe mide la búsqueda defensiva de la aprobación social y la escala de Taylor pretende medir la sintomatología ansiosa en general.

- Escala de pensamientos depresógenos y ansiógenos intrusivos de Clark et al. (1985). Esta escala busca medir frecuencia, tristeza inducida, preocupación, la dificultad de "sacarse de la cabeza» y la desaprobación asociadas a seis pensamientos ansiógenos y seis pensamientos depresógenos.

- Escala de cogniciones (CCL). Se construyó a partir de los pensamientos automáticos de pacientes ansiosos y depresivos con intención de discriminar entre ambos estados.

\section{PROGRAMAS DE INTERVENCIÓN EDUCATIVA BASADOS EN EL DESARROLLO EMOCIONAL DE LA INTELIGENCIA}

No existe un único tipo de intervención, incluyendo a la intervención emocional, capaz de resolver todas las situaciones de prevención, desarrollo o corrección que pueden manifestarse en los ámbitos educativos.

Prestar atención al desarrollo emocional de los escolares es una necesidad añadida que debería integrarse. de forma programada, en el currículum, con el resto de las acciones educativas que pretenden mejorar los procesos de enseñanza-aprendizaje y prevenir aparición de conflictos.

El marco de la LOGSE propicia este tipo de intervenciones cuando resalta, en la educación obligatoria, el perfil social sobre el cognitivo en los escolares; la necesidad de preparar para ejercer en la vida valores que precisan de 
un adecuado desarrollo emocional y propone como objetivo fundamental lograr la máxima autonomía personal en los procesos de aprendizaje, lo que confiere una gran importancia al desarrollo equilibrado de las emociones y a la motivación de sí mismo para seguir aprendiendo.

Ello supone adquirir e integrar, durante la etapa de formación obligatoria, modelos de aprendizaje válidos y permanentes que, una vez integrados a nivel cognitivo y emocional, puedan ser utilizados por las personas en su desarrollo de vida.

A continuación exponemos una síntesis de programas de educación emocional que podrían utilizarse integrándolos en los currículum escolares, extraídos de Goleman, D. (1997, pp. 402 y ss.).

\section{${ }^{*}$ El currículum de Self Science}

Objetivos: Educación de los sentimientos, tanto los propios como los que se corresponden con el ámbito de las relaciones. Elevar el nivel de competencia emocional, desarrollo social, habilidades vitales, aprendizaje social y emocional. Cooperación y trabajo colaborativo. Evitación de conflictos y resolución de desacuerdos, control de las emociones.

Componentes fundamentales:

- Conciencia de uno mismo: observarse a sí mismo y reconocer sus propios sentimientos; elaborar un vocabulario de los sentimientos; conocer las relaciones existentes entre los pensamientos, los sentimientos y las reacciones.

- Toma de decisiones personales: examinar las propias acciones y conocer sus consecuencias; saber si una determinada decisión está gobernada por el pensamiento o por el sentimiento; aplicar esta comprensión a temas tales como el sexo y las drogas.

- Dominar los sentimientos: "charlar con uno mismo» para comprender los mensajes negativos, como las valoraciones negativas de uno mismo; comprender lo que se halla detrás de un determinado sentimiento (por ejemplo, el dolor que subyace a la ira); buscar formas de manejar el miedo, la ansiedad, la ira y la tristeza.

- Manejar el estrés: aprender el valor de ejercicios tales como la imaginación guiada y los métodos de relajación.

- Empatía: comprender los sentimientos y las preocupaciones de los demás y asumir su perspectiva; darse cuenta de las diferentes formas en que la gente siente las cosas. 
- Comunicaciones: desarrollar la capacidad de hablar de los sentimientos; aprender a escuchar y a hacer preguntas; distinguir entre lo que alguien hace o dice y sus propias reacciones o juicios al respecto; enviar mensajes desde el «yo» en lugar de hacerlo desde la censura.

- Apertura: valorar la apertura y la confianza en las relaciones; reconocer cuándo puede uno arriesgarse a hablar de los sentimientos más profundos.

- Intuición: identificar pautas en su vida y en sus reacciones emocionales y reconocer pautas similares en los demás.

- Autoaceptación: sentirse bien consigo mismo y considerarse desde una perspectiva positiva; reconocer sus propias fortalezas y debilidades; ser capaz de reírse de sí mismo.

- Responsabilidad personal: asumir la responsabilidad; reconocer las consecuencias de sus decisiones y de sus acciones; aceptar sus sentimientos y sus estados de ánimo; perseverar en los compromisos adquiridos (por ejemplo, estudiar).

- Asertividad: afirmar sus intereses y sentimientos sin ira ni pasividad.

- Dinámica de grupo: cooperación; saber cuándo y cómo mandar y cuándo obedecer.

- Solución de conflictos: aprender a jugar limpio con los compañeros, padres y maestros; aprender el modelo ganador de negociar compromisos.

\section{* Evaluaciones de programas de aprendizaje social y emocional}

- Proyecto de desarrollo infantil

Eric Schaps, Developmental Studies Center, Oakland, California.

Evaluación realizada en escuelas de California del Norte (grado K-6) y valorada por observadores independientes comparando los resultados obtenidos con escuelas normales que sirvieron como grupo de control.

Resultados:

- más responsables

- más asertivos

- más populares y sobresalientes 
- más prosociales y colaboradores

- mejor comprensión de los demás

- más considerados e interesados

- más estrategias prosociales para la solución de problemas interpersonales

- más armoniosos

- más «democráticos»

- más hábiles de resolución de conflictos.

- PATHS (caminos)

Mark Greenberg, Fast Track Project, Universidad de Washington.

Evaluación realizada en escuelas de Seattle (grados del 1 al 5) y valorada por maestros comparando alumnos de un grupo de control con tres tipos de grupos experimentales: 1) estudiantes regulares, 2) estudiantes sordos y 3) estudiantes de educación especial.

Resultados:

- mejora en habilidades cognitivas sociales

- mejora en emoción, reconocimiento y comprensión

- mayor autocontrol

- mejor planificación para la resolución de tareas cognitivas

- más pensamiento antes de actuar

- resolución más eficaz de conflictos

- clima más positivo en clase.

Estudiantes con necesidades especiales

Mejor conducta en clase en:

- tolerancia a la frustración

- habilidades sociales asertivas 
- habilidades con los compañeros

- participación

- sociabilidad

- autocontrol.

Mejora en la comprensión emocional.

- reconocimiento

- etiquetado

- disminución de la tristeza y la depresión (según autoinformes)

- disminución del grado de ansiedad y aislamiento.

- Proyecto de desarrollo social de Seattle

J. David Hawkins, Social Development Research Group, Universidad de Washington.

Evaluación realizada en escuelas elementales y secundarias por observadores independientes y utilizando criterios objetivos, en comparación con escuelas en las que no se llevó a cabo el programa y que sirvieron como grupo de control.

Resultados:

- vínculos más positivos con la familia y la escuela

- chicos menos agresivos y chicas menos autodestructivas

- menos índice de suspensos y expulsiones entre estudiantes de bajo rendimiento

- menor iniciación al uso de las drogas

- menos delincuencia

- mejores puntuaciones en test de logro estandarizados.

- Programa de promoción de la competencia social Yale-New Haven

Roger Weissberg, Universidad de Illinois en Chicago. 
Evaluación realizada en las escuelas públicas de New Haven (grados 5 al 8) y valorada por observadores independientes, e informes de los alumnos y los maestros comparándolos con un grupo de control.

Resultados:

- mejora en habilidades de solución de problemas

- más colaboración con los compañeros

- mayor dominio de los impulsos

- mejora de la conducta

- mejora de la eficacia y de la popularidad interpersonal

- aumento de la capacidad de afrontar las situaciones

- más habilidad para manejar los problemas interpersonales

- mejor control de la ansiedad

- menos conductas delictivas

- mejora en las habilidades de resolución de conflictos.

- Programa de resolución creativa de conflictos.

Linda Lantieri, National Center for Resolving Conflict Creatively Program (una iniciativa de Educators for Social Responsability), New York City.

Evaluación realizada en escuelas de New York City (grado K-12) y valorada por maestros antes y después de la realización del programa.

Resultado:

- menos violencia en clase

- pocas agresiones verbales en clase

- clima más amable

- mayor disposición para cooperar

- más empatía

- mejora en habilidades de comunicación. 
- Proyecto de desarrollo de la conciencia social: aprendizaje de resolución de problemas sociales.

Maurice Elias, Universidad de Rutgers.

Evaluación realizada en escuelas de New Jersey (grado K-6) y valorada por maestros, compañeros de clase y registros escolares, comparándolos con alumnos que no participaron en el proyecto.

Resultados:

- mayor sensibilidad a los sentimientos de los demás

- mejor comprensión de las consecuencias de su conducta

- mayor capacidad para "valorar» las situaciones interpersonales y planificar acciones adecuadas

- mayor autoestima

- más conducta prosocial

- buscar la ayuda de los compañeros

- transición más adecuada a la escuela secundaria

- menor conducta antisocial, autodestructiva y socialmente desordenada, aun cuando fuera seguida de ingreso en el instituto

- aumento de la capacidad de aprender a aprender

- más autocontrol, mayor conciencia social y mejor toma de decisiones sociales tanto dentro como fuera del aula.

\section{CONSIDERACIONES FINALES}

Hemos querido, en lo expuesto, resaltar dos ideas principales:

La explicación de comportamiento inteligente a partir de planteamientos cognitivos no es suficiente para elaborar pautas de intervención educativa para el desarrollo integral de los aprendices. Se hace necesaria una concepción, según Coll (2001: 129), "más amplia y plural de la inteligencia que supere la centración, común a ambas perspectivas (refiriéndose a la diferencial psicométrica y la de procesamiento de la información), en lo que podemos llamar "inteligencia académica escolar" y explore en profundidad la diversidad de capacidades que pueden estar implicadas en el comportamiento inteligente". 
La teoría de inteligencias múltiples desarrollada por Gardner y apoyada por los trabajos de la últimas décadas de Seternberg (1997), unida a las nuevas explicaciones de los comportamientos inteligentes que parten de la Psicología social, Psicología cultural..., según Coll (2001), van a subrayar el carácter social, contextual y cultural de la actividad inteligente. Por tanto, nos sitúan en otra dimensión de la persona en relación a sí misma y a los demás, en la cual es más fácil, a la vez que necesaria, la comprensión global de la persona y su contexto, y nos ofrece matices del desarrollo social y emocional en los que la educación, desde las edades más tempranas, puede provocar en los aprendices mayor autorregulación de sus relaciones, en aras de su equilibrio personal, y mayor motivación en sus logros escolares. Como afirma Miras (2001: 311): «Si acordamos que los procesos escolares de aprendizaje y enseñanza son procesos que necesariamente implican a las personas de una forma global, parece lógico que, del mismo modo que es importante considerar los distintos factores que definen la capacidad y los recursos cognitivos del alumno frente a un determinado proceso de aprendizaje - sus habilidades, estrategias, conocimientos especificos-, parece conveniente interrogarse también respecto a la diversidad de factores que determinan las restantes capacidades con las que el alumno se enfrenta a dicho proceso, en especial sus capacidades emocionales y de equilibrio personal».

\section{BIBLIOGRAFÍA}

COLL, C. (1991). Concepción constructivista y planteamiento curricular. Cuadernos de Pedagogía, 188. 8-11.

COLl, C., PALACIOA, J., MARCHESI, A. (2001). Desarrollo Psicológico y Educación: Psicología de la Educación Escolar. Madrid. Alianza Editorial.

DELVAL J. (1990): Los fines de la educación. Madrid. México. Siglo XXI.

GARDNER, H. y HATCH, T. (1989). «Multiple Intelligences Go to School». Educational Research, 18,8.

GARDNER, H. (1993). Multiple Intelligence: The Theory in Practice. Nueva York. Basic Books.

GENOVARD C. y CASTELLÓ A. (1990). El limite superior: Aspectos psicopedagógicos de la excepcionalidad intelectual. Madrid. Pirámide.
GOLEMAN, D. (1997). Inteligencia Emocional. Barcelona. Kairos

KABAT-ZINN. J. (1994). Wherever You Go, There You Are. Nueva York. Hyperion.

MARTÍN, D. y BOECK, K. (1997). ¿Qué es inteligencia emocional? Madrid. EDAF.

MARTINEAU, S. y ENGELHART, D. (1997). El test de inteligencia emocional. Barcelona. Martínez Roca.

MIRAS, M. (2001) : Afectos, emociones, atribuciones y expectativas: el sentido del aprendizaje escolar. En Coll, C. Palacios, J y Marchesi, A. (2001). Desarrollo Psicológico y Educación: Psicología de la Educación Escolar. Madrid. Alianza Editorial.

SALOVEY, P. y MAYER, J.D. (1990). «Emotional Intelligence». Imagina- 
tion, Cognitión and Personality, 9, pp. 185-211.

STERNBERG, R.J. (1985). La inteligencia práctica y las habilidades personales. Nueva York. Cambridge University Press.

TORRABADELLA, P. (1997). Cómo desarrollar la inteligencia emocional. Barcelona. Oasis S.I.
VERGARA, A. y otros (1989). "Problemas generales de la medición de estados afectivos y de operacionalización de los diferentes conceptos (Estado de ánimo, frecuencia, intensidad y variabilidad). En Echebarría A. y Páez D. Emociones: Perspectivas psicosociales, pp. 473 y ss. Madrid. Fundamentos.

\section{RESUMEN}

El artículo trata de describir las diferentes concepciones que se han tenido de la inteligencia desde el campo de la psicología y de la educación para llegar a la consideración, en estos últimos años, de nuevos conceptos, el de inteligencias múltiples e inteligencia emocional, fundamentales en el desarrollo óptimo de las personas a lo largo de la vida.

Son numerosos los estudios sobre competencia social, autocontrol de las emociones, autoeficacia..., que han evidenciado las repercusiones que puede tener en ese desarrollo óptimo la inteligencia emocional, ligada tanto a la competencia cognitiva como a los aspectos citados, es un constructo dinámico que se puede modificar y desarrollar. Adquiere un carácter necesario en la educación formal, la cual se debe encargar no solo del desarrollo intelectual, sino de un desarrollo integral del individuo y prepararlo para la vida.

Palabras clave: Modelos de Inteligencia, Inteligencia Emocional, Indicadores, Instrumentos de apreciación, Orientación Educativa.

\section{ABSTRACT}

The article tries to describing the different conceptions that be have had of the intelligence from the field of the psychology and of the education to arrive to the consideration, in these last years of new concepts, that of multiple intelligences and emotional intelligence, fundamental in the optimum development of the persons throughout the life. 
They are numerous the studies on social competition, autocontrol of the emotions, autoeficacia, that they have evidenced the repercussions that they can have in that optimum development the emotional intelligence, bound to the competition cognitiva as well as to the cited aspects, it is a constructo dynamical that it can be modified and developed. Acquires a necessary character in the formal education, the one which it should be to entrust not alone del intellectual development, but with an integral development of the individual and to prepare it for the life.

Key words: Models of Intelgencia, Emotional Intelligence, Indicative, appraisal Instruments, Educational Direction. 